\title{
ReaR

\section{Nuevas recomendaciones sobre el uso de corticoides en el paciente crítico}

Artículo original: Djillali Annane, Stephen M. Pastores, Bram Rochwerg, Wiebke Arlt, Robert A. Balk, Albertus Beishuizen, et al. Guidelines for the diagnosis and management of critical illness-related corticosteroid insufficiency (CIRCI) in critically ill patients (Part I and II). Society of Critical Care Medicine (SCCM) and European Society of Intensive Care Medicine (ESICM) 2017 (HTML)

De la Calle Gil I, Carvajal Revuelta E, García Álvarez R

Hospital Universitario 12 de Octubre. Madrid.

\section{Resumen}

La utilización de corticoides en el paciente crítico es una práctica clínica habitual, aunque no siempre respaldada por la evidencia científica. Los datos aportados por estas nuevas guías intentan responder a algunas de las dudas que se nos plantean diariamente en el ejercicio de nuestra actividad.

\section{Introducción}

La utilización de corticoides en el paciente crítico es una práctica clínica habitual, aunque no siempre respaldada por la evidencia científica. Los datos aportados por estas nuevas guías intentan responder a algunas de las dudas que se nos plantean diariamente en el ejercicio de nuestra actividad.

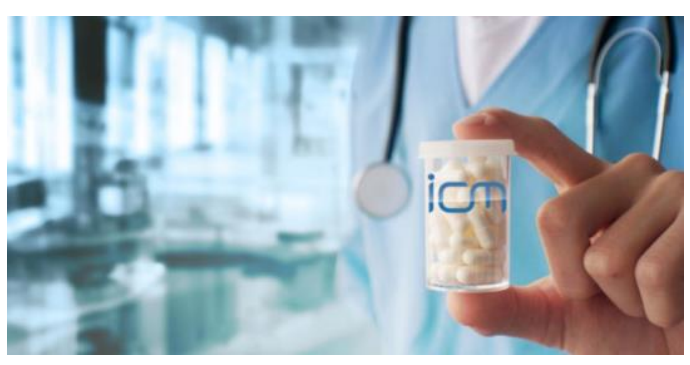

La respuesta inflamatoria sistémica es un mecanismo altamente organizado de nuestro organismo desencadenado ante una amenaza, ya sea infecciosa o no, cuya finalidad es el mantenimiento de la homeostasis. Sin embargo, es necesario que esta respuesta sea regulada de forma precisa, tanto en intensidad como en duración, para poder ser beneficiosa.

Se describió en 2008 el concepto CIRCI (Critical illness-related corticosteroids insufficiency) introducido por un grupo de trabajo internacional de la SCCM (Society of Critical Care Medicine) y de la Sociedad Europea de Cuidados Intensivos, que describe la disfunción del eje hipotálamo-hipofisario en la respuesta al estrés en el paciente crítico. Está caracterizada por una alteración de la respuesta inflamatoria sistémica, que determina una inadecuada actividad antiinflamatoria mediada por glucocorticoides a nivel intra $y$ extracelular.

En función de la población estudiada y los criterios diagnósticos considerados, la incidencia de CIRCI ha sido estimada en un $10-20 \%$, alcanzando valores del $60 \%$ en el shock séptico. Esta condición está relacionada con una mayor estancia en unidades de críticos y una elevada morbimortalidad. Por ello, es primordial la detección precoz de los pacientes en riesgo de desarrollar CIRCI y su 
diagnóstico correcto una vez establecida para poder focalizar la intervención terapéutica a llevar a cabo y evitar el sobretratamiento de individuos no afectos.

\section{Material y Métodos}

Las recomendaciones (guías CIRCI) son una actualización de las recomendaciones de 2008 basadas en los hallazgos más recientes a partir de una revisión sistemática de estudios entre 2008 y 2017. Estas recomendaciones están formuladas en base a la metodología GRADE (Grading of Recommendations Assessment, Development, and Evaluation).

La intensidad de cada recomendación fue clasificada como fuerte $o$ condicional. En cuanto a la calidad de la evidencia, inicialmente se graduó desde elevada a muy baja, basada en el diseño individual del estudio: alta para ensayos controlados randomizados y baja para estudios observacionales.

Posteriormente se subgraduó según los siguientes factores: el riesgo de sesgos, la consistencia de los resultados y la claridad y precisión de la evidencia.

La aprobación de cada recomendación requirió la conformidad de al menos el $80 \%$ de los miembros del grupo de trabajo. Se tuvieron en cuenta las siguientes cuestiones al formular las recomendaciones: calidad de la evidencia, balance entre efectos deseables de los principios y preferencias del paciente asociadas a la decisión, los recursos requeridos e igualdad en su aplicación, la aceptación por parte de los proveedores de salud y la factibilidad de su implementación. El objetivo principal de este estudio fue actualizar las recomendaciones en el diagnóstico y manejo de la insuficiencia corticoidea en el paciente crítico
(CIRCI), adulto y pediátrico. No debe ser interpretada como un estándar único de manejo, sino como unas pautas a considerar en la práctica clínica diaria en función del paciente a tratar.

\section{Resultados}

Por lo que respecta al diagnóstico de CIRCI, el grupo de trabajo no fue capaz de determinar un único test de referencia, aunque el delta cortisol tras la administración de ACTH (cosyntropin, $250 \mu \mathrm{g}$ ) definido como un cambio en los niveles basales de cortisol a los 60 minutos $<9 \mu \mathrm{g} / \mathrm{dl}$ y un valor aleatorio de cortisol plasmático $<10$ $\mu \mathrm{g} / \mathrm{dl}$ pueden usarse en la práctica clínica habitual. No recomiendan el uso del cortisol libre en plasma o cortisol salival (nivel de evidencia bajo-muy bajo).

En lo que se refiere al tratamiento, en función de la condición específica por la que se desarrolla el CIRCI:

- No recomiendan la administración de corticoesteroides en pacientes sépticos en ausencia de shock.

- Recomiendan el uso de hidrocortisona intravenosa $<400 \mathrm{mg} /$ día durante $\geq 3$ días a dosis plenas en pacientes con shock séptico que no responden a fluidoterapia ni a dosis moderadaselevadas de vasopresores.

- Recomiendan el uso de metilprednisolona intravenosa 1 $\mathrm{mg} / \mathrm{kg} /$ día en pacientes con síndrome de distrés respiratorio agudo (SDRA) precoz (hasta el $7^{\circ}$ día desde su inicio) moderado-severo ( $\mathrm{PaO} 2 / \mathrm{FiO} 2<200)$; y en SDRA tardío (después del $6^{\circ}$ día de inicio), metilprednisolona intravenosa 2 $\mathrm{mg} / \mathrm{kg} / \mathrm{d}$ ía; seguidos ambos de un descenso paulatino durante 13 días. Se recomienda metilprednisolona porque penetra mejor en el tejido pulmonar y permanece más tiempo. 
- No recomiendan el uso de corticoesteroides en pacientes con trauma severo.

- En neumonía adquirida en la comunidad, recomiendan el uso de corticoides durante 5-7 días a una dosis diaria de menos de $400 \mathrm{mg}$ de hidrocortisona intravenosa.

- Recomiendan no usar corticoides en la gripe.

- Recomiendan el uso de corticoides en meningitis bacteriana, en pacientes con circulación extracorpórea y en pacientes que han sufrido parada cardíaca.

\section{Discusión}

Por lo que respecta a su diagnóstico, recomiendan el uso del test de estimulación con $\mathrm{ACTH}$, pero en las unidades de críticos de nuestro medio su uso ya no está extendido. Además, la Surviving Sepsis Campaign (SSC), ya en sus últimas tres ediciones $2,3,4$, no recomienda su uso sistemático. Por otro lado, los estudios clínicos CORTICUS e HYPRESS muestran una respuesta clínica similar a corticoides independientemente del resultado del test.

Las recomendaciones terapéuticas se centran en condiciones específicas de las que hablamos a continuación:

- Hasta el momento no existe ningún tratamiento que mejore la supervivencia en pacientes con sepsis, aunque el seguimiento de las recomendaciones de la SSC3 ha demostrado una disminución global de la mortalidad ${ }^{6}$.

Ambas guías, SSC3 y CIRCI, recomiendan la utilización de hidrocortisona en pacientes con shock séptico, aunque difieren en la dosis y forma de administración (en SSC3 la recomendación es en perfusión continua a dosis de $200 \mathrm{mg}$ al día, sólo tras una adecuada resucitación y con necesidad de vasopresores a dosis crecientes). Recientemente, con posterioridad a la publicación de estas guías CIRCI, se ha publicado el ensayo clínico ADRENAL5, en el que no se ha observado aumento de la supervivencia con el uso de corticoides en pacientes con shock séptico, aunque sí un menor número de días de ventilación mecánica, más rápida resolución del shock y menos necesidad transfusión de hemoderivados. Los propios autores de estas guías CIRCI (que desconocían los resultados del ensayo ADRENAL) reconocen que sus propias recomendaciones podrían variar a la luz de los resultados de este último estudio.

- En lo que se refiere al SDRA, los corticoides continúan generando controversia. Los estudios en los que se apoya su uso son controvertidos debido a una serie de limitaciones ${ }^{7,8}$ como: gran diversidad de etiologías responsables del SDRA, falta de consenso en la clasificación de los pacientes según su severidad, inicio del tratamiento en estadios diferentes de la enfermedad, dosis y duración del tratamiento, etc. Por lo que hasta ahora no existía un consenso acerca de uso.

Estas guías CIRCI recomiendan su uso en fase precoces y tardías, algo que entra en contradicción con lo recomendado hasta ahora, que sólo consideraba su uso en fases iniciales; además el descenso debe ser paulatino para que no haya efecto inflamatorio de rebote. Esta recomendación está basada fundamentalmente en un estudio de Meduri9 publicado recientemente y que ha demostrado beneficios en la supervivencia y disminución en la duración de la ventilación mecánica. Creemos que en los próximos años veremos estudios que apoyarán o rebatirán esta nueva recomendación. 
- En cuanto al trauma severo, no se recomienda el uso dado los potenciales efectos adversos e incluso un posible aumento de mortalidad.

- Por lo que respecta a la neumonía adquirida en la comunidad (NAC): recomiendan el uso de corticoides a toda NAC, sin especificar su gravedad, cuando en la mayoría de estudios se recoge que el mayor beneficio es en NAC grave ${ }^{10}$. En las guías previas de 2008, no se recogía esta recomendación.

- Dada la ausencia de ensayos clínicos aleatorizados relacionados con gripe y corticoides, recomiendan no usar corticoides en esta situación. En los estudios observacionales relativos ${ }^{11}$, se concluye un posible aumento de la mortalidad asociada a su administración considerando como potenciales factores de confusión las características basales individuales $y$ el momento de administración (posible efecto favorable en fases tardías, sin repercutir en la respuesta defensiva inicial del hospedador y tras el control de la replicación viral por el tratamiento antiviral).

- Las indicaciones de las guías CIRCI recomiendan el tratamiento corticoideo en la meningitis bacteriana pero dejan varios aspectos importantes sin aclarar: la duración mínima del tratamiento (siendo el régimen de dexametasona 0,4-0,6 $\mathrm{mg} / \mathrm{Kg} /$ día en dosis diaria durante 4 días el más empleado en los ensayos clínicos incluidos en una revisión sistemática de la Cochrane ${ }^{12}$ ), el tipo de corticoide empleado (al parecer la dexametasona presenta mayor penetración y vida media en líquido cefalorraquídeo respecto a metilprednisolona e hidrocortisona), la administración antes, simultáneamente o después de la primera dosis de antibioterapia intravenosa y los efectos a largo plazo de su uso (siendo la recurrencia de la fiebre el único efecto adverso demostrado y siendo este de carácter concurrente a la enfermedad aguda y no a largo plazo).

- En pacientes que han sufrido parada cardíaca recomiendan, con un bajo nivel de evidencia, el uso de corticoides (sin especificar tipo ni dosis) dado que parece que aumenta la supervivencia y reduce las secuelas neurológicas. Los estudios 13, 14 que demuestran estos beneficios tienen ciertas restricciones debido a que la resucitación cardiopulmonar no es homogénea, el tipo y dosis de corticoide difiere, al igual que los cuidados tras la parada cardiaca. No obstante, fisiológicamente parece lógico que en una situación de bajo gasto con hipoperfusión hacia la corteza suprarrenal se reduzca la producción de corticoides endógenos y que su aporte externo ayude a mantener un correcto tono vascular $\mathrm{y}$ mejore además la acción de drogas vasoactivas.

- Aunque en las guías CIRCI se recomienda el uso de corticoides en la circulación extracorpórea (CEC) con un nivel de evidencia moderado, son varios los estudios que ponen de manifiesto que la reducción de la mortalidad 15, 16, 17 y de la aparición de fibrilación auricular 16,17 no es estadísticamente significativa.

\section{Conclusión}

La utilización de corticoides en el paciente crítico es una práctica clínica habitual, aunque no siempre respaldada por la evidencia científica. Los datos aportados por estas nuevas guías intentan responder a algunas de las dudas que se nos plantean diariamente en el ejercicio de nuestra actividad. En cuanto a las recomendaciones que recogen, nos parece que lo más novedoso sería el uso de corticoides en SDRA tardío. Los mismos autores reconocen que la evidencia a menudo es 
baja y que se requieren ensayos clínicos adicionales.

\section{Bibliografía}

1. Djillali Annane, Stephen M. Pastores, Wiebke Arlt, Robert A. Balk, Albertus Beishuizen, et al. Critical illness-related corticosteroid insufficiency (CIRCI): a narrative review from a Multispecialty Task Force of the SCCM and ESICM. (PubMed) (HTML)

2. Rhodes A, Evans LE, Alhazzani W, Levy MM, Antonelli M, Ferrer R. Surviving sepsis campaign: international guidelines for management of sepsis and septic shock: 2016. Critical Care Med 2017; 45 (3): 486-552. (PubMed)

3. Dellinger RP, Levy MM, Rhodes A, Annane D, Gerlach H, Opal SM. Surviving sepsis campaign: international guidelines for management of sepsis and septic shock: 2012. Critical Care Med 2013 Feb; 41(2): 580-637. (PubMed)

4. Dellinger RP, Levy MM, Carlet JM, Bion J, Parker MM, Jaeschke R. Surviving sepsis campaign: international guidelines for management of sepsis and septic shock: 2008. Critical Care Medicine 2008 Jan;36(1):296-327. (PubMed)

5. Venkatesh B, Finfer S, Cohen J,Rajbhandari $\mathrm{D}$,Arabi $\mathrm{Y}$, Bellomo $\mathrm{R}$, et al. Adjunctive glucocorticoid therapy in patients with Septic Shock. ADRENAL Trial Investigators and the Australian-New Zealand Intensive Care Society Clinical Trials Group. N Engl J Med. 2018 Jan 19. (HTML)

6. Herrán Monge R, Muriel Bombín A, García García MM, Merino García PA, Martínez Barrios M, Andaluz D, et al. Epidemiology and Changes in Mortality of Sepsis after the Implementation of Surviving Sepsis, Campaign Guidelines. J. Intensive Care Med 2017 Jan. (PubMed) ( $\underline{\text { HTML + PDF) }}$

7. Ranieri VM, Rubenfeld GD, Thompson BT, Ferguson ND, Caldwell E, Fan E, et al. Acute respiratory distress syndrome: the Berlin definition. JAMA 2012 Jun 20;307 (23):252633. (PubMed)

8. Ruan SY, Lin HH, Huang ChT, Kuo PH, Wu $\mathrm{HD}, \mathrm{Yu}$ CJ. Exploring the heterogeneity of effecs of corticosteroids on acute respiratory distress syndrome: a systematic review and meta-analysis. Critical Care 2014,18:R63. ( $\underline{\text { PubMed }}$ ( HTML) (

9. Meduri GU, Bridges L, Shin MC, Marik PE, Siemieniuk RAC, Kocak Met, al. Prolonged glucocorticoid treatment is associated with improved ARDS outcomes: analysis of individual patients' data from four randomized trials and trial-level metaanalysis of the updated literature. Intensive Care Med (2016) 42(5):829-840. ( PubMed)

10. Stern A, Skalsky K, Avni T, Carrara E, Leibovici L, Paul M. Corticosteroids for pneumonia. Cochrane Database of Systematic Reviews. 2017, 12.

11. Delaney JW, Pintor R, Long J, Lamontagne F, Adhikari NK, Kumar A, et al. and on behalf of the Canadian Critical Care Trials Group H1N1 Collaborative. Delaney et al. The influence of corticosteroid treatment on the outcome of influenza A (H1N1pdm09)-related critical illness. Critical Care (2016) 20:75. (

12. Brouwer MC, McIntyre P, Prasad K, van de Beek D. Corticosteroids for acute bacterial meningitis. Cochrane Database of Systematic Reviews 2013, Issue 6. Art. No.: CD004405. (PubMed) (Abstract)

13. Tsai MS, Chuang PY, Yu PH, Huang $\mathrm{CH}$, Tang CH, Chang WT, et al. Glucocorticoid use during cardiopulmonary resucitation may be beneficial for cardiac arrest. International Journal of Cardiology 2016 Nov 1;222:629-35. (PubMed)

14. Botnaru T, Altherwi T, Dankoff J. Improved neurologic outcomes after cardiac arrest with combined administration of vasopressin, steroids, and epinephrine compared to epinephrine alone. CJEM. 2015 Mar;17(2):2025. (PubMed) (Abstract)

15. Whitlock RP, Chan S, Devereaux PJ, Sun J, Rubens FD, Thorlund K, et al. Clinical benefit of steroid use in patients undergoing cardiopulmonary bypas: a meta-analysis of randomized trials. Eur Heart J 2008;29: 2592600. (PubMed) (HTML) (HTML2)

16. Dieleman JM, Nierich AP, Rosseel PM, van der Maaten JM, Hofland J, Diephuis JC, et al. Intraoperative high-dose dexamethasone for cardiac surgery: a randomized controlled trial. 
JAMA 308:1761-1767. 2012. (PubMed) (HTML)

17. Whitlock RP, Devereaux PJ, Teoh $\mathrm{KH}$, Lamy A, Vincent J, Pogue J, et al. Methylprednisolone in patients undergoing cardiopulmonary bypass (SIRS): a randomised, double-blind, placebo-controlled trial. Lancet 386(10000):1243-1253.2015. (PubMed)
Correspondencia al autor

Isabel De la Calle Gil

isabel.calle.gil@gmail.com

Médico Residente. Unidad de Cuidados Intensivos de Anestesia.

Hospital Universitario 12 de Octubre. Madrid.

Aceptado para blog en junio de 2018. 